\title{
Fasting Has Limited Impacts on the Result of Penicillin Allergy Skin Test: An Observation Study
}

\author{
XIONG Zhaohui ${ }^{1}$; WU Wenrong ${ }^{1}$; HUANG Xuanjie ${ }^{1 *}$;WU Wenrong ${ }^{1}$; NI Xiaomin ${ }^{1}$ \\ ${ }^{1}$ The Seventh Affiliated Hospital of Sun Yat-sen University, Shenzhen, Guangdong Province, \\ China, 518000. \\ ${ }^{*}$ HUANG Xuanjie is the corresponding author. https://orcid.org/0000-0002-8811-7937
}

Article History Received 14 June 2021 Accepted 25 July 2021 Published 30 September 2021 Cite this Article XIONG Zhaohui; WU Wenrong; HUANG Xuanjie;WU Wenrong; NI Xiaomin. Fasting Has Limited Impacts on the Result of Penicillin Allergy Skin Test: An Observation Study [J].Medical Research, 2021.3(3):9-14, http://dx.doi.org/mrhk/10.6913/MRHK.202109_3(3).0002 Copyright @ 2021 Creative Publishing Co., Limited. All rights reserved.Email: mrhk@mrhk.cc

\begin{abstract}
Objective Penicillin allergy skin test is a common procedure in nursing care. Many patients can only do an allergy skin test on fasting due to the disease condition. However, whether or not the fasting state has any effects on the skin testing results remains unclear. Therefore, this study aimed to determine the effects of fasting on the results of penicillin allergy skin tests.

Methods This observational cohort study analyzed the clinical data of 200 patients undergoing a penicillin skin test in the emergency center of The Seventh Affiliated Hospital of Sun Yat-sen University from August 1, 2020, to December 31, 2020, of which 100 cases were fasting patients and 100 were non-fasting patients. The information including gender, age, allergy, skin test results, dizziness, and gastrointestinal symptoms were collected and compared.
\end{abstract}

Result Among the 200 patients, five patients (2.5\%) tested positive for the penicillin-allergy skin tests. Three patients (3\%) tested positive in the fasting group, while two patients $(2 \%)$ in the non-fasting group tested positive (Relative Risk: 1.5 ; $95 \%$ Confidence Interval: 0.3055 to 7.397; $\mathrm{P}=0.65)$.

Conclusion These data suggest that fasting may have limited impacts on the results of penicillin allergy skin tests.

Keywords Fasting; Penicillin skin test; Results; Observation analysis

\section{INTRODUCTION}

Penicillin has been a powerful weapon for treating bacterial infectious diseases in human history since it was first discovered by the Scottish scientist, Alexander Fleming, in $1928^{[1]}$. Despite its important clinical value and broad-spectrum antibacterial effects, about $10 \%$ of the population report that they are allergic to penicillin. Penicillin allergic responses are a type of hypersensitivity response mediated by $\operatorname{IgE}$ or $\mathrm{T}$ lymphocytes ${ }^{[2,3]}$. Severe allergic reactions 
could occur in about $0.03 \%$ of the general population ${ }^{[3]}$. Therefore, in China and many other countries, most patients must undergo a penicillin allergy skin test before using it ${ }^{[4-6]}$.

Penicillin allergy skin test is a common procedure in nursing clinics. A positive skin reaction indicating that the patient is truly allergic to penicillin is an itchy, red bump lasting around 30 minutes and resolves later, with or without other systemic symptoms such as dizziness, palpitation, and nausea. Before the testing, the patient's allergy history, medication history, family history, eating status, and psychological status are needed to be evaluated ${ }^{[7]}$. However, in the clinic, many patients can only undergo allergy skin tests on fasting due to their illness condition; and whether the fasting state has any effects on the skin testing results is still unclear. Therefore, in the current study, we performed a cohort study on 200 patients who underwent penicillin skin test with or without fasting to determine the effect of fasting on skin testing results.

\section{METHODS}

\section{Patient information}

Two hundred patients undergoing penicillin skin test in the emergency center of The Seventh Affiliated Hospital of Sun Yat-sen University from August 1, 2020, to December 31, 2020, were enrolled and divided into the fasting group $(n=100)$ and non-fasting group $(n=100)$ according to their eating status. The inclusion criteria were that the patients voluntarily participated in the study and signed an informed consent form. The exclusion criteria were as followed: 1) patients with a history of fainting blood and needles; 2) patients who were critically ill and could not cooperate; 3) patients taking antihistamine drugs or receiving hormone therapy within 7 days. Eventually, 100 fasting patients and 100 non-fasting patients were included. There were 45 males and 55 females in the fasting group with ages between 9 and 64 years old (mean \pm standard deviation: $35.98 \pm 12.227$ ). There were 42 males and 58 females in the non-fasting group, with ages ranging from 11 to 65 years old (mean \pm standard deviation: $37.98 \pm 11.56$ ). The baseline information, such as age and gender, were comparable between the two groups of patients ( $>00.05)$.

\section{Penicillin allergy skin test}

Both groups used the penicillin sodium produced by Guangzhou Baiyunshan Tianxin Pharmaceutical Co., Ltd. The batch number was 190653, and the dose per bottle was 800,000 units. The penicillin preparation solution was $0.9 \%$ saline for injection, produced by China Otsuka Pharmaceutical Co., Ltd. (10ml each). The syringes $(1 \mathrm{ml}$ and $5 \mathrm{ml})$ were produced by Shanghai Kangdelai Enterprise Development Group Co., Ltd. The configuration method was carried out according to the 50 Nursing Operation Techniques in Guangdong Province.

The details of the preparation of the penicillin skin test solution are listed below.

1. Disinfect the opening of the penicillin bottle according to the specifications; inject $4 \mathrm{ml}$ of $0.9 \%$ saline into a bottle of penicillin sodium and shake it well (the concentration of penicillin sodium is $200,000 \mathrm{u} / \mathrm{ml}$ ).

2. Use a $1 \mathrm{ml}$ syringe to take $0.1 \mathrm{ml}$ stock solution in the penicillin bottle, add $0.9 \mathrm{ml}$ of $0.9 \%$ saline to dilute to $1 \mathrm{ml}$ and shake it well (the concentration of penicillin sodium 
is $20,000 \mathrm{u} / \mathrm{ml})$.

3. Then discharge $0.9 \mathrm{ml}$ of the test solution in the syringe, and then continue to dilute with $0.9 \mathrm{ml}$ of $0.9 \%$ saline and shake it well (the concentration of penicillin sodium is $2,000 \mathrm{u} / \mathrm{ml})$.

4. Discharge $0.9 \mathrm{ml}$ of the test solution in the syringe, continue to dilute with $0.9 \mathrm{ml}$ of $0.9 \%$ saline again and shake it well (the concentration of penicillin sodium is $200 \mathrm{u} / \mathrm{ml}$ ). Therefore, the final skin test solution concentration is $200 \mathrm{u} / \mathrm{ml}$. Take $0.1 \mathrm{ml}$ of the final skin test solution for the skin test.

The details of penicillin skin test procedures are listed below.

1. The skin test was performed on the two groups of patients according to the routine nursing operation, and the injection volume was $0.1 \mathrm{ml}$ of the penicillin skin test solution prepared as described above (20 units).

2. Before the skin test, the patient's medication history, allergy history, family history, eating status, mental state, illness, and psychological state were evaluated, and the skin test injection was performed on the inner side of the lower forearm of the patient.

3. All patients were instructed not to touch or scratch the injection site.

4. After 20 minutes, the results of the skin test were judged by two nurses.

5. The judgment standard of the skin test result was based on the consensus of experts in the penicillin skin test ${ }^{[4]}$, as listed below.

Table 1. Evaluation of penicillin allergy skin test results.

\begin{tabular}{lll}
\hline Tests & Manifestation (injection site of the skin) & Systemic symptoms \\
\hline \multirow{2}{*}{ Negative } & No change in size, no redness, swelling, or No symptoms or apparent \\
& flushing around. & discomforts. \\
& The bump is enlarged and flushed with a Dizziness, flustered, \\
Positive & diameter greater than $1 \mathrm{~cm}$; Pseudopods around nausea, and even \\
& the bump could be observed, accompanied by anaphylactic shock may
\end{tabular}

Note: The negative result indicates that the patient is not allergic to penicillin. The positive result indicates that the patient is allergic to penicillin.

\section{Statistical analysis}

The SPSS22.0 statistical software was used to perform the statistical analysis. The Chi-Squared test was used for data analysis. The p-value smaller than 0.05 indicated the difference was statically significant. 


\section{RESULTS}

\section{Fasting had limited effects on the results of penicillin allergy skin tests.}

As shown in Table 2, among the 200 patients, five patients (2.5\%) tested positive for the penicillin allergy skin tests. Three patients $(3 \%)$ in the fasting group were positive, while two patients $(2 \%)$ in the non-fasting group tested positive (Relative Risk: 1.5; 95\% Confidence Interval: 0.3055 to $7.397 ; \mathrm{P}=0.65$ ). These results indicate that penicillin allergy skin test results were comparable between the fasting group and the non-fasting group. Therefore, the effect of fasting on the penicillin-allergy skin test may be limited.

Table 2. Results of penicillin allergy skin tests in the fasting and non-fasting groups

\begin{tabular}{llll}
\hline Groups & Positive cases & Negative cases & Total \\
\hline Fasting & 3 & 97 & 100 \\
Non-fasting & 2 & 98 & 100 \\
Total & 5 & 195 & 200 \\
\hline
\end{tabular}

Chi-square test: Relative Risk (RR): 1.5; 95\% Confidence Interval (CI): 0.3055 to 7.397; $\mathrm{P}=0.65 ; \mathrm{Z}=0.45$.

\section{DISCUSSION}

In this study with 200 research participants, our data confirmed that the positive rate in the penicillin allergy skin tests was very rare $(2.5 \%)$. The comparable positive rate between the two groups (fasting: $3 \%$ vs non-fasting: $2 \%, \mathrm{P}=0.65$ ) suggested that the effect of fasting on the penicillin allergy skin test results might be limited. This information may be helpful for clinical workers.

The sixth edition of "Basic Nursing Science" of People's Press pointed out that patients should not take skin tests when they are fasting. The reason is that when the individuals are fasting, skin injections can cause dizziness, nausea and other reactions, which are easily confused with symptoms of allergic reactions. Therefore, fasting during skin tests may be not conducive to the judgment of the testing results. However, the 2017 edition of the Penicillin Skin Test Expert Consensus does not stipulate that patients undergoing penicillin skin tests need to be performed in a non-fasting state. Therefore, whether fasting can affect the skin test results remains to be addressed.

In terms of the immune mechanisms ${ }^{[8]}$, drug allergic reactions refer to the adverse reactions of patients with idiosyncratic constitutions after using a certain drug, also known as allergic reactions. Studies have shown ${ }^{[4,8]}$ that penicillin-allergic reactions are type I allergic reactions, mainly mediated by IgE in the body, causing anaphylactic shock, urticaria, laryngeal edema and bronchial asthma.

Jialin Guo et al. ${ }^{[8]}$ has reported that three factors could cause penicillin allergy. The first factor is drug quality. The quality and purity of the drug directly affect the incidence of allergic reactions. The higher the purity of the drug, the lower the incidence of allergic 
reactions. The second is the influence of drug metabolites. The occurrence of allergic reactions requires the combination of hapten and protein carriers to form a complete antigen and sensitize the body. This process is an irreversible covalent bond. Under normal circumstances, the drug itself is not easy to form the above-mentioned combination. Only the metabolites of some chemically active drugs can be combined with protein or polysaccharide carriers in the body, such as commonly used antibacterial drugs, antipyretic analgesics, or vaccines, etc. The third is patient-derived factors, including genetic factors and allergy physique. Different strains of the same animal and different individuals can produce different intensities of immune responses to the same antigen. For example, those with genetically secreted immunoglobulin A deficiency will have increased permeability of the digestive tract mucosa, undigested or incompletely digested proteins. Allergens enter the body and can cause allergic reactions. Regarding allergy physique, the individual's body reactivity changes due to endocrine disorders and enzyme deficiencies, which could lead to allergic reactions ${ }^{[9]}$. In addition, the occurrence of allergic reactions is also related to the patient's age, gender, health status, stress stimulation, metabolic disorders, etc. However, in this study, our data supported that whether the patient is fasting has limited effects on the results of the penicillin skin test.

We acknowledge that this study has several shortcomings. First, this study is an observational study, and it has not been randomized. Second, the sample size of the study is small, so the test power may be insufficient. More large-scale studies are needed to further prove this conclusion.

\section{SUMMARY}

This observational study supports that fasting has limited impacts on the results of penicillin allergy skin tests.

\section{ACKNOWLEDGMENT}

None.

\section{STATEMENT}

There is no conflict of interest in this article.

\section{REFERENCES}

1 Bennett JW, Chung KT. Alexander Fleming and the discovery of penicillin. Adv Appl Microbiol. 2001;49:163-84. doi: 10.1016/s0065-2164(01)49013-7.

2 Shenoy ES, Macy E, Rowe T, Blumenthal KG. Evaluation and Management of Penicillin Allergy: A Review. JAMA. 2019 Jan 15;321(2):188-199. doi: 10.1001/jama.2018.19283.

3 Gonzalez-Estrada A, Radojicic C. Penicillin allergy: A practical guide for clinicians. Cleve Clin J Med. 2015 May;82(5):295-300. doi: 10.3949/ccjm.82a.14111.

4 The National Health and Family Planning Commission of the People's Republic of China (NHFPC). Expert consensus on penicillin skin test [J]. National Medical Journal of China, 2017(40): 3143-3146.doi:10.3760/cma.j.issn.0376-2491.2017.40.005.

5 Dong-fang Zhao, Hui-zhi Zhang, Suo-di Zhai, Fan Li, Yu-jia Zhang. Clinical practice 
guidance of penicillin skin test. Clinical Medication Journal. 2020,18(10):54-57. doi: 10.3969/j.issn. 1672-3384.2020.10.012.

6 Ping Jia, Caixia Xie, Yamin Zhu, Xiaoqun Li.Investigation on the implementation of the skin test of penicillin drugs [J]. China Medicine and Pharmacy,2020,10(22):174-176+186. doi: 10.3969/j.issn.2095-0616.2020.22.049.

7 Pongdee T, Li JT. Evaluation and Management of Penicillin Allergy. Mayo Clin Proc. 2018 Jan;93(1):101-107. doi: 10.1016/j.mayocp.2017.09.020.

8 Jialin Guo, Nana Zhang, Yuanyuan Gong et al. Research progress on immunological mechanism of allergic reactions caused by antibacterial drugs. Evaluation and Analysis of Drug-Use in Hospitals of China. 2015. 15(01): 140-143. doi:10.14009/j.issn. 1672-2124.2015.01.046.

9 Chunrong Sun. Causes of drug allergic reactions and nursing countermeasures [J]. China Modern Medicine, 2008,15(23):118-119. doi: 10.3969/j.issn. 1674-4721.2008.23.081. 